\title{
The Small Town Endowment Plan in The Coordinated Development of Beijing-Tianjin-Hebei View : Shunping County in Hebei Province Example
}

\author{
Shuai Lia ${ }^{a}$, Hongwei Li
}

1Urban and Rural Construction College, Agricultural University of Hebei, Baoding, China

a $\underline{532599162 @ q q . c o m}$

Key words: The Coordinated Development of Beijing-Tianjin-Hebei; Small Town; Endowment Plan; Shunping county, Hebei province

Abstract. In the face of ageing population and coordinated development strategy of the BeijingTianjin-Hebei region, Beijing-Tianjin-Hebei region endowment development of small town exists the following problems: endowment mode single, existing polarization between regions, low level of endowment services, inadequate planning, etc. Taking Shunping county in hebei province as an example, this paper puts forward small towns multi-factors coordination endowment planning strategy of Beijing-Tianjin-Hebei region. That is the industry system building and rendering guide, diversificate pattern building, space hierarchical layout, facilities' regional coordination, institutionalizad implementation of security. Hope it can provide reference for other small towns in Beijing-Tianjin-Hebei region.

\section{Introduction}

According to the sixth national census data, in 2010 Beijing-Tianjin-Hebei region 65-year-old and above people has reached 8.73 million, the proportion of the total population is $8.36 \%{ }^{[1]}$. The elderly population in Beijing-Tianjin-Hebei area is very big. The "getting old before getting rich" problem is prominent. May 2015, "Beijing-Tianjin-Hebei collaborative development planning outline" completed formally. It has brought great opportunities and challenges for the planning and development of Beijing-Tianjin-Hebei , especially endowment planning of Tianjin-Hebei small town.

This article starting with the current situation of Beijing-Tianjin-Hebei endowment services, analyses and summarise new requirements of Beijing-Tianjin-Hebei endowment planning, putted forward by Beijing-Tianjin-Hebei collaborative development this major national strategy. And Taking Shunping County in Hebei Province as an example, this paper discusses the strategy of Beijing-Tianjin-Hebei small town endowment plan in the context of this strategy, in order to play a certain role in the development and research of the endowment facilities planning in the future.

\section{The current situation of the endowment industry of Beijing-Tianjin-Hebei}

Currently there are three major problems in the situation of the endowment industry of BeijingTianjin-Hebei region : (1) The development level of endowment institutions is low, it's total amount is not enough and it's quality is not high. The three areas are not coordinated. According to the statistics to the end of 2012, Beijing-Tianjin-Hebei region have endowment bedspace average 24.27 per thousands of people, only near to international per 1000 people 50 beds, also lower than the level of some developing countries per thousand people 30 beds (as in Table 1 below). (2) Endowment policy is different in the there region, and the level of agency services is low. In 2016 per capita basic endowment for urban and rural residents in Hebei province was 80 yuan / month, while Beijing and Tianjin two cities in 2015, reached 470 yuan / month and 245 yuan / month ${ }^{[2]}$. Investment of policy funds is small and specialized endowment service team are scarce. 
Table 1 Endowment institutions statistics

\begin{tabular}{|c|c|c|c|}
\hline & Beijing & Tianjin & Shijiazhuang \\
\hline $\begin{array}{c}\text { The number of elderly population ratio (above } \\
60 \text { years old) }\end{array}$ & $20.30 \%$ & $18.90 \%$ & $14.71 \%$ \\
\hline $\begin{array}{c}\text { Total number of beds in the elderly } \\
\text { Public bed }\end{array}$ & 76172 & 38490 & 30323 \\
\hline Private bed & 36603 & 4475 & 18375 \\
\hline Endowment agency & 400 & 29672 & 368 \\
\hline Endowment institutions (public) & 212 & 339 & 256 \\
\hline Endowment institutions (private) & 188 & 140 & 112 \\
\hline Number of beds per thousand elderly & 29 & 20.5 & 20.67 \\
\hline
\end{tabular}

Note: Data from Which city is the most responsible for the elderly. Oriental Outlook Weekly, 2013.45.

\section{The issues and challenges about small town endowment development in Beijing-Tianjin- Hebei}

With the accelerated development process of the coordinated development of Beijing-Tianjin-Hebei, it has brought opportunities and challenges for the endowment of small towns. However, due to the large environment of endowment industry, so there are many problems in the current development of small towns.

\subsection{The endowment pattern is single and desire for change}

At present, endowment pattern of small towns is still based on family, endowment institutions and medical endowment pattern combined with the auxiliary ${ }^{[3]}$. But the family endowment pattern is easily to be affected by the physical condition of family members, and the combination of institutional care and medical support is less to estimate the level of spiritual needs of the elderly.

\subsection{The development is not coordinated and the polarization between the regions is serious}

As of the end of 2013, the number of rural household registration of the elderly population in Beijing is 575 thousand, and can reach rural average 83 beds per thousand people. Hebei is 5 million 828 thousand and 400 , and the average per thousand beds indicators are only 21.15. Hebei rural household registration elderly have a number of only 1/4 of Beijing, and did not meet international standards.

\subsection{The endowment service level is low}

The endowment service level is low, and the urgent need to attract professional talents ${ }^{[4]}$. The vast majority of qualified social service workers choose to work in the big city where have high pay and good living conditions. This caused the lack of talents in small towns.

\subsection{Endowment plan is inadequate, the depth is to be strengthened}

Endowment plan is inadequate, the depth is to be strengthened. Compared with the urban endowment plan, the urban planning and construction of small towns in the area are mainly based on the overall planning of small towns (the overall planning) and rural construction planning.

In view of the above problems, taking Shunping county of Hebei province as an example, this paper introduces the development of the endowment industry, the spatial distribution of endowment facilities and the specific planning measures about the small towns of Beijing-Tianjing-Hebei. I hope it can provide a reference for other small town's endowment plan and construction. 


\section{The endowment planning of Shunping county, Hebei}

\subsection{General situation of Shunping county, Hebei}

Shunping county is located in the west of Hebei province, southwest of Baoding. It is the transition zone of Taihang Mountains to the North China Plain. It also is in the central zone of the big economic circle of Beijing, Tianjin, Shijiazhuang, Baoding, Taiyuan, as shown in figure 1. Shunping is rich in tourism resources, historical culture and natural landscape. It has a wealth of development potential.
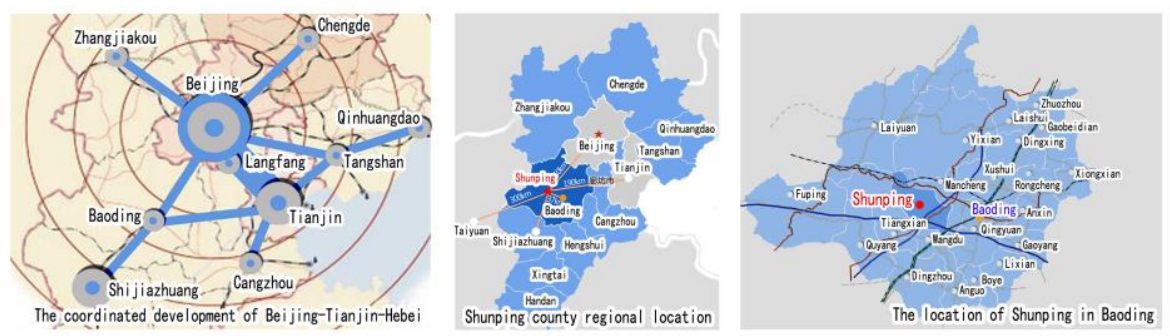

Figure 1. Shunping County bitmap

\subsection{Multi factor coordinated small town endowment planning strategy}

\subsubsection{The endowment industry system construction and whole county guidance}

In May 2016, Hebei Province issued the "health and medical industry development plan (20162020)", which established around the Beijing and Tianjin health care industry, and strived to build a green ecological requirements of health care and elderly maintenance base. The elderly of BeijingTianjin continue to the influx of Shunping. The number of elderly population will continue to increase the scale of its consumption, and more elderly groups according to their own needs to experience the life of endowment services. The proportion of high-end endowment service product demand (consumption object mainly for older age groups in Beijing and Tianjin) will continue to rise, so it will form a endowment service industry, while covering the apartment accommodation, medical care, catering, tourism consumption, costume jewelry, culture, entertainment, and investment banking and other areas of industry value chain ${ }^{[5]}$.

Using primary, secondary and tertiary industries and the endowment industry combined to achieve the whole county spatial layout of endowment industry, namely, to guide the endowment industry to expand to the entire region.

Primary industry is mainly combined with "the Hebei Province Shunping County overall planning of urban and rural (2013-2030)" (hereinafter referred to as the version of the 2013 general rules) the arrangement planning of urban and rural industry in the construction of organic agriculture demonstration area requirements and Yi Qi Shan village carnivals rural tourism development leading, to guide the elderly people to participate in leisure and sightseeing agriculture, eco agriculture picking, rural cultural experience related to agricultural tourism activities, the elderly tourism consumption, catering services, culture and entertainment projects to the entire region expand.

The second industry mainly with the construction of the economic development demonstration zone in Shunping County, in the introduction of the enterprise level, it is inclined to the endowment development, elderly health care products, daily necessities of the elderly etc. Third industry focus to do a good job of leisure tourism, catering, leasing and other services, and targetly arrangements related facilities.

Through the construction of the endowment industry system and the whole county industrialization, it will finally realize the county endowment industry, industrial endowment, the 
perfect harmony into three times industry and endowment industryendowment industry and urban industrial symbiosis development pattern of mutually beneficial symbiosis.

\subsubsection{The diversified construction of endowment pattern}

The selection of small town endowment pattern is affected by the elderly population, the ecological environment, the number of facilities and other related factors. Shunping county as a part of the carrier about the evacuation function of Beijing faces the dual pressures of its own endowment and external endowment.

For its oun elderly population, it mainly uses the family endowment pattern. Institutions endowment pattern is an auxiliary. It explores the pattern of medical support combined. In the face of external elderly group of Beijing-Tianjin, Shunping county should actively explores the new pattern of the housing endowment, community endowment and remote interactive endowment.It can solve the pressure from the external elderly group. Through this program it can be perfect to build a diversified endowment pattern.

\subsubsection{Level layout of the endowment space}

The level of the layout of the endowment space should be considered from the overall planning (general rules), special planning and regulatory detailed planning (control rules) and planned guidance.

General planning level: It is mainly to carry on the classification, the hierarchical layout to guide. First of all, through general layout that the elderly in nursing homes (county), homes for the elderly (township) - old station (Village) layout planning makes up their own old-age short board. Secondly, through the combination of local culture, tourism and other resources, it will construct of four endowment industry cities, as shown in Figure 2.

They merge the local culture. It has a strong external bearing capacity. The organic combination of general and special two aspects of layout, realize the rationalization and the spatial layout of the pension level.

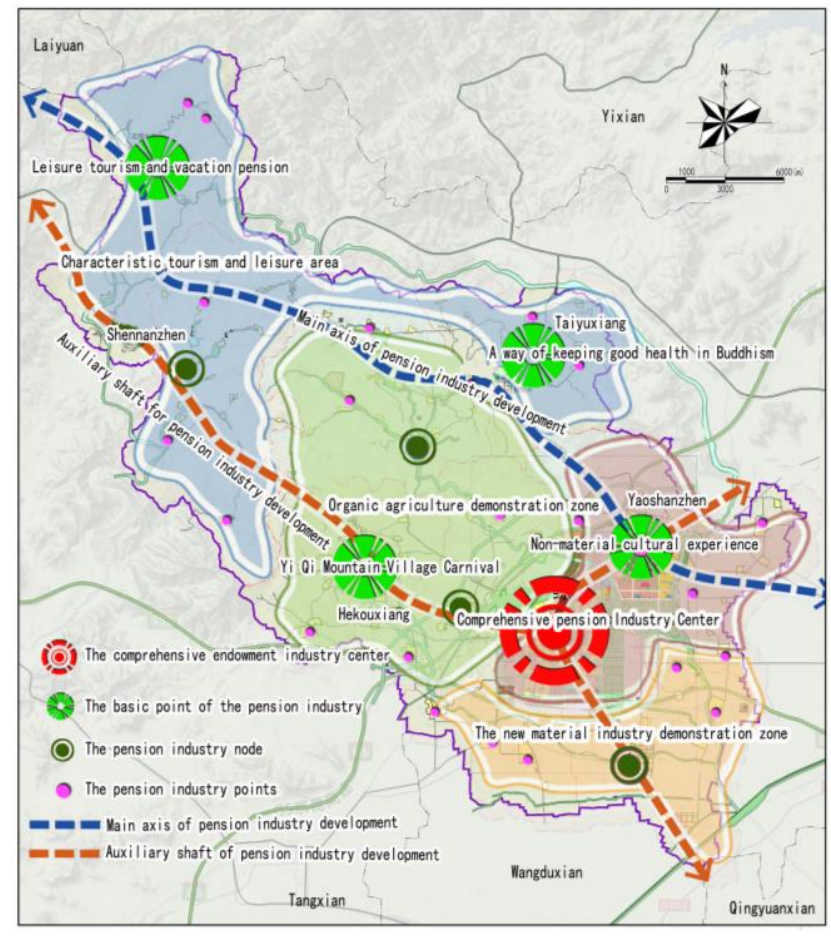

Figure 2 Pension industry layout plan 
Special planning level: It is mainly to make up the loopholes and improve the general layout. Control planning level: It is mainly used for the implementation of the endowment plan to determine the use of the land. It controls the relevant mandatory and recommended indicators, such as the barrier free design of the interior environment. The determination of the index is mainly based on Code of City and Town facilities for the Aged Planning \& Design and other related standards. According to the actual situation it can be adjusted on the develop strength, so as to realize land intensive $\mathrm{e}^{[6]}$.

\subsubsection{The area cooperation of endowment facilities}

In this paper, through access to the standard of endowment facilities in Beijing-Tianjin-Hebei, found that the basic standard of endowment facilities is different. There is a lack of coordination between the regions. At present, the unified standards of small town endowment facilities and combing is very necessary. Shunping county endowment facilities configuration has been readjusted in the planning, as shown in table 2 . But it also needs to be verified on the spot.

Table 2 Endowment facilities allocation standard

\begin{tabular}{|c|c|c|c|c|}
\hline \multirow{2}{*}{ Project } & \multicolumn{4}{|c|}{ The standards of the allocation } \\
\cline { 2 - 5 } & \multicolumn{2}{|c|}{ General scale $\left.\mathbf{( m}^{\mathbf{2}}\right)$} & \multicolumn{2}{c|}{ Indicator per thousand people $\left.\mathbf{( m}^{\mathbf{2}}\right)$} \\
\cline { 2 - 5 } & The building area & Land area & The building area & Land area \\
\hline $\begin{array}{c}\text { Elderly nursing } \\
\text { home }\end{array}$ & $4000-5000$ & $5000-6000$ & 80 & 120 \\
\hline $\begin{array}{c}\text { Endowment industry } \\
\text { city }\end{array}$ & -15000 & 60 & 1000 \\
\hline $\begin{array}{c}\text { Home of respect for } \\
\text { the aged }\end{array}$ & $\geq 900$ & $\geq 1200$ & & \\
\hline $\begin{array}{c}\text { Elderly activity } \\
\text { station }\end{array}$ & $\geq 150$ & $\geq 200$ & & \\
\hline
\end{tabular}

\subsubsection{Institutionalizad implementation of security}

Small towns endowment development pattern dealing with the Beijing-Tianjin-Hebei collaborative development strategy should have qualified institutional safeguards. In order to ensure the good endowment system is builded effectly, mainly from the policy support, operations management, talent introduction and training of three aspects to carry out.

Policy support aspect: governments at all levels need to clearly know the endowment will face pressure, conscious of the small endowment policy inclination; Operations management aspect: to break the limited manpower and financial resources of small towns, this paper argues that the development pattern of the related enterprises dominate the endowment program development and government regulation are necessary, such as four endowment industry city projects of Shunping county. Talent introduction and training aspect: increasing financial investment, introducting talent of has the domestic advanced nursing concept and technology class talents and related endowment product research and development of scientific research, On the base, cultivate the local talent, and enrich the endowment industry talent pool.

\section{Conclusion}

At present, the research on pension planning more focused on the cities, in towns pensions is less, and discussed in the coordinated development of Beijing-Tianjin-Hebei is few. Based on shunping county in hebei province as an example, this paper expounds the strategy pension planning of small towns under the background of coordinated development of Beijing-Tianjin-Hebei, but the exemplary role for other small towns has not demonstrate. The industry system building and rendering guide, diversificate mode building, space hierarchical layout, facilities, regional coordination, institutionalizad implementation of security putted forward bythis article, are 
response to the coordinated development of Beijing-Tianjin-Hebei, and population aging acceleration period time background such. Because pension industry is a professional industry, involving every aspect of society and economy, so all the pension problems cannot be expounded comprehensivly and professionally in this article, but this article still expect to plays a certain reference value to other small towns pension planning of Beijing-Tianjin-Hebei region.

\section{References}

[1] Liu Li. The Building Design of Beijing-Tianjin-Hebei endowment service system[J]. Journal of Cooperation in Economy and Technology, 2014, 22:174-175.

[2] Sun Jianfu Dai Luwei. The environment ,objectives and principles of coordinated development of Beijing-Tianjin-Hebei endowment services [J]. Economic Research Reference, 2015,63: 4146.

[3] Li Shuang. Research on planning and design for endowment facilities insmall towns: Taking the town of Hengdao Hezi as case study[D]. Harbin institute of technology,2013.

[4] Xu Zhixin. To accelerate the development of pension services in Hangzhou [J]. Policy outlook, 2011,06:41-43.Shuang.

[5] Chen Xiaohui,Yang Hongping. Urban-Rural Planning in the Context of Population Aging: A Case Study on Jiangsu Province [J].City Planning Review, 2013,09:17-21.

[6] Shi Yu,Jiang Ruyuan. The Endowment Plan in the Area View: Taking Jiande City Sandu Town as Example[J]. Planner,2015,S1:118-122. 